\title{
Effects of Correlated Shadowing: Connectivity, Localization, and RF Tomography
}

\author{
Neal Patwari and Piyush Agrawal \\ Sensing and Processing Across Networks Lab \\ ECE Department, University of Utah, Salt Lake City, USA \\ [npatwari, pagrawal] dece.utah.edu
}

\begin{abstract}
Unlike current models for radio channel shadowing indicate, real-world shadowing losses on different links in a network are not independent. The correlations have both detrimental and beneficial impacts on sensor, ad hoc, and mesh networks. First, the probability of network connectivity reduces when link shadowing correlations are considered. Next, the variance bounds for sensor self-localization change, and provide the insight that algorithms must infer localization information from link correlations in order to avoid significant degradation from correlated shadowing. Finally, a major benefit is that shadowing correlations between links enable the tomographic imaging of an environment from pairwise RSS measurements. This paper applies measurement-based models, and measurements themselves, to analyze and to verify both the benefits and drawbacks of correlated link shadowing.
\end{abstract}

\section{Introduction}

This paper addresses the effects of an accurate radio layer model on multi-hop wireless network performance analysis. In particular, we apply a path loss model which connects link shadowing losses to the physical environment in which the network operates. Because nearby links are often affected by the same shadowers in the environment, link losses can be correlated. The new probability model for path loss improves the accuracy of analysis of the effects of the physical layer in multi-hop networks. In this paper, we quantify the effects of correlated shadowing in three topics: network connectivity (Section 3), sensor received signalstrength (RSS)-based localization bounds (Section 4), and radio tomographic imaging (RTI) (Section 5). This quantification uses simulation and analysis using a proposed link shadowing model, and experimental measurements.

Each section introduces its topic in more detail, but in short, existing i.i.d. link fading models overestimate network connectivity and do not permit complete analysis of RSS self-localization lower bounds on variance. Further, the same effect which causes correlated shadowing also enables the imaging of environments using link measurements of RSS, which may lead to the development of wholebuilding imaging sensor networks for security applications or for emergency use by fire-fighters and police.

This paper presents the first broad study of the effects of link fading correlations on sensor, ad hoc, and mesh network performance. We first, in this section, discuss existing path loss and fading models for multi-hop networks, and give intuitions as to why they are not adequate. Section 2 provides an overview of the correlated link fading model used in this paper, the network shadowing (NeSh) model, which was reported in [2]. The lack of a validated model has prevented past research from quantifying link correlation effects. Sections 3 through 5 present the effects of the model in the three areas mentioned above, and Section 6 concludes and discusses open research topics.

\subsection{Path Loss and Fading}

The path loss on a link has three contributions: (1)Largescale path loss due to distance; (2)Shadowing loss due to obstructions; and (3)Non-shadowing loss due to multipath (a.k.a. small-scale or frequency selective fading). Given this delineation, we denote $P_{i, j}$ as the measured received power at node $j$ transmitted by node $i$,

$$
\begin{aligned}
P_{i, j} & =\bar{P}\left(d_{i, j}\right)-Z_{i, j}, \\
Z_{i, j} & =X_{i, j}+Y_{i, j},
\end{aligned}
$$

where $d_{i, j}$ is the distance between nodes $i$ and $j, \bar{P}(d)$ is the ensemble mean $\mathrm{dBm}$ received power at distance $d, X_{i, j}$ is the $\mathrm{dB}$ shadowing loss, and $Y_{i, j}$ is the non-shadow fading loss in $\mathrm{dB}$. We refer to $Z_{i, j}$ as the total fading loss. Ensem- 
ble mean received power at distance $d$ is given by [9],

$$
\bar{P}(d)=P_{T}-\Pi_{0}-10 n_{p} \log _{10} \frac{d}{\Delta_{0}},
$$

where $P_{T}$ is the transmitted power in $\mathrm{dBm}, n_{p}$ is the path loss exponent, and $\Pi_{0}$ is the loss experienced at a short reference distance $\Delta_{0}$ from the transmitter antenna.

\subsection{Existing Models}

The literature commonly considers two fading models. The first is that fading is insignificant, i.e., $Z_{i, j}=0$ for all links $(i, j)$. This is commonly referred to as the circular coverage model, because links in all directions will be disconnected deterministically for $d$ such that $\bar{P}(d)$ exceeds a threshold. Very significant analytical results have been derived using the circular coverage model regarding connectivity (as reviewed in [14]) and capacity [8], to note two example applications. The circular coverage model is a common assumption in published research [13].

The second model is that fading $Z_{i, j}$ is random, independent and identically distributed on each link. We refer to this as the i.i.d. link fading model, and it is used in analysis of many wireless networks, and has been used in published analysis and simulation of multi-hop networks [10,3].

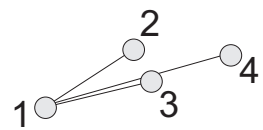

\section{Figure 1. Three links with a common node.}

In reality, neither model accurately represents the radio channel for multi-hop networks. While coverage area is certainly not circular, it generally has some continuous shape. The i.i.d. link fading model does not have any spatial memory, so there is no sense of coverage area. For example, consider nodes which can communicate with node 1 in Figure 1 . Node 2 may be disconnected while node 3 is connected, or vice versa, regardless of how close nodes 2 and 3 are to each other. Also, the fading on links $(1,3)$ and $(1,4)$ are independent, even though objects which attenuate link $(1,3)$ probably also attenuate link $(1,4)$. These disconnects between the i.i.d. link fading model and reality motivate the application of a model with link shadowing correlation. Perhaps the best motivation is that link shadowing correlations have been experimentally observed in measured sensor networks [2], yet their impacts have not yet been studied.

\section{Network Shadowing Model}

In order to develop analysis and simulations which consider correlated link shadowing, we must apply a statistical model which has been verified using measurements. In mobile radio networks, the model of Gudmundson [7] is used to model shadow fading correlations for a mobile link with a common base station. However, this model is unable to model correlations between links with no common endpoints, as are common in ad hoc networks.

In this paper, we apply the network shadowing (NeSh) model of [2]. To model the experimentally observed characteristic of correlated link shadowing, the network shadowing (NeSh) model first models the environment in which the network operates. It then considers shadowing losses to be a function of that environment, which has the effect of correlating losses on geographically proximate links.

The shadowing caused by an environment is quantified in an underlying spatial loss field $p(\mathbf{x})$. The NeSh model assumes that $p(\mathbf{x})$ is an isotropic wide-sense stationary Gaussian random field with zero mean and exponentially decaying spatial correlation. Specifically,

$$
E\left[p\left(\mathbf{x}_{i}\right) p\left(\mathbf{x}_{j}\right)\right]=R_{p}\left(d_{i, j}\right)=\frac{\sigma_{X}^{2}}{\delta} e^{-\frac{d_{i, j}}{\delta}}
$$

where $d_{i, j}=\left\|\mathbf{x}_{j}-\mathbf{x}_{i}\right\|$ is the Euclidian distance between $\mathbf{x}_{i}$ and $\mathbf{x}_{j}, \delta$ is a space constant and $\sigma_{X}^{2}$ is the variance of $X_{i, j}$.

Next, the NeSh model formulates the shadowing on link $a=(i, j)$ as a normalized integral of the $p(\mathbf{x})$ over the line between link endpoints $\mathbf{x}_{i}$ and $\mathbf{x}_{j}$,

$$
X_{a} \triangleq \frac{1}{d_{i, j}^{1 / 2}} \int_{\mathbf{x}_{i}}^{\mathbf{x}_{j}} \mathbf{p}(\mathbf{y}) d \mathbf{y} .
$$

Single Link Properties: The NeSh model agrees with two important empirically-observed link shadowing properties:

Prop-I The variance of link shadowing is approximately constant with path length [9],[21].

Prop-II Shadowing losses are Gaussian [21],[18].

The model in (5) can be seen to have Prop-II, since $X_{a}$ is a scaled integral of a Gaussian random process. To show Prop-I, we note that $E\left[X_{a}\right]=0$, and

$$
\operatorname{Var}\left[X_{a}\right]=\frac{1}{d_{i, j}} \int_{\mathbf{x}_{i}}^{\mathbf{x}_{j}} \int_{\mathbf{x}_{i}}^{\mathbf{x}_{j}} R_{p}(\|\boldsymbol{\beta}-\boldsymbol{\alpha}\|) d \boldsymbol{\alpha}^{T} d \boldsymbol{\beta} .
$$

Using (4) as the model for spatial covariance,

$$
\operatorname{Var}\left[X_{a}\right]=\sigma_{X}^{2}\left[1-\frac{\delta}{d_{i, j}}\left(1-e^{-d_{i, j} / \delta}\right)\right] .
$$

When $d_{i, j}>>\delta$, the NeSh model has Prop-I,

$$
\operatorname{Var}\left[X_{a}\right] \approx \sigma_{X}^{2} .
$$

Joint Link Properties: Given two links $a, b \in \mathbb{Z}^{2}$, both $X_{a}$ and $X_{b}$ are functions of the same shadowing field $p(\mathbf{x})$, thus 
the model (5) introduces correlation between them. The covariance between $X_{a}$ and $X_{b}$ is,

$$
\operatorname{Cov}\left(X_{a}, X_{b}\right)=\frac{\sigma_{X}^{2} / \delta}{d_{i, j}^{1 / 2} d_{l, m}^{1 / 2}} \int_{\mathbf{x}_{i}}^{\mathbf{x}_{j}} \int_{\mathbf{x}_{l}}^{\mathbf{x}_{m}} e^{-\frac{\|\boldsymbol{\beta}-\boldsymbol{\alpha}\|}{\delta}} d \boldsymbol{\alpha}^{T} d \boldsymbol{\beta}
$$

The covariance (9) is computed by numerical integration, using code available on [1].

\subsection{Non-Shadow Fading}

As given in (1), received power $P_{i, j}$ consists of losses due both to shadow fading $X_{i, j}$ and non-shadow fading $Y_{i, j}$. The NeSh model assumes that $\left\{Y_{i, j}\right\}_{i, j}$ are independent. Nodes in ad hoc, sensor, and mesh networks are typically separated by many wavelengths, and small-scale fading correlation is approximately zero at such distances [5]. Further, the NeSh model assumes that small scale fading is independent of shadow fading.

In particular, $Y_{i, j}$ is modeled as Gaussian (in $\mathrm{dB}$ ) with zero mean and variance $\sigma_{Y}^{2}$. While small scale fading for narrowband fading channels is typically modeled as Rayleigh, Rician, or other non-Gaussian distribution, the $\mathrm{NeSh}$ model assumes that nodes have a wideband receiver which effectively averages narrowband fading losses across frequencies in its bandwidth. As an average of small-scale fading losses at many frequencies, $Y_{i, j}$, by a central limit argument, is approximately Gaussian.

\subsection{Joint Link Received Power Model}

On a single link $a=(i, j)$, total fading loss $Z_{a}=X_{a}+$ $Y_{a}$. Since $X_{a}$ and $Y_{a}$ are independent and Gaussian (in dB), total fading $Z_{a}$ is also Gaussian with variance

$$
\sigma_{d B}^{2} \triangleq \operatorname{Var}\left[Z_{a}\right] \approx \sigma_{X}^{2}+\sigma_{Y}^{2}
$$

Furthermore, we consider the joint model for the total fading on all links in the network. Let $a_{1}, \ldots, a_{K}$ for $a_{k}=$ $\left(i_{k}, j_{k}\right)$ be an enumeration of the $K$ unique measured links in the network. A unique link $a_{k}$ must have both that $a_{k} \neq$ $a_{l}$ for all $l \neq k$ and that the reciprocal link is not included, i.e., $\left(j_{k}, i_{k}\right) \neq a_{l}$ for all $l$. The vector RSS on all links is

$$
\mathbf{P}=\left[P_{a_{1}}, \ldots, P_{a_{K}}\right] .
$$

The vector $\mathbf{P}$ is multivariate Gaussian, and thus it is completely specified by its mean and covariance. We define vector $\overline{\mathbf{P}}$ as the mean of $\mathbf{P}$, which is given by

$$
\overline{\mathbf{P}}=\left[\bar{P}\left(d_{a_{1}}\right), \ldots, \bar{P}\left(d_{a_{K}}\right)\right],
$$

where $\bar{P}\left(d_{a_{k}}\right)=\bar{P}\left(d_{i_{k}, j_{k}}\right)$ is given in (3). Next, denoting $C$ to be the covariance matrix of $\mathbf{P}$,

$$
C_{k, l}=\sigma_{Y}^{2} I_{k, l}+\operatorname{Cov}\left(X_{a_{k}}, X_{a_{l}}\right)
$$

where $I_{k, l}=1$ if $k=l$ and 0 otherwise, and $\operatorname{Cov}\left(X_{a}, X_{b}\right)$ is given in (9).

\subsection{Research Evidence}

The NeSh model was tested in a measurement campaign in [2] which measured fifteen realizations of indoor wireless sensor networks. Each realization was measured in a randomly-altered environment. Following the measurements, the losses on each 'link geometry', that is, the relative positioning of two links, were used to calculate a covariance between the shadowing on two links. The model explained $80.4 \%$ of the measured covariances on the tested link geometries.

Both current i.i.d. link fading models and the NeSh model require two parameters, $\sigma_{d B}^{2}$ and $n_{p}$ [21]. The NeSh model requires two additional parameters, $\sigma_{X}^{2}$ and $\delta$. In [2], these are reported to be $\sigma_{X}^{2} / \sigma_{d B}^{2}=0.29$ and $\delta=0.21 \mathrm{~m}$. These measured parameters in all of the following analysis and simulation.

\subsection{Model Limitations}

We note that the NeSh model has limitations. First, it is a purely statistical model, not a solution to the electromagnetic wave propagation equation. To simulate a network deployed in a known place with known obstructions, one could use ray-tracing to determine loss on each link with high accuracy. The NeSh model is valuable only for simulations of random deployments.

Second, the model in [2] was tested for 2-D, not 3-D deployments. Although not tested, the model is applicable to 3-D; one could apply 3-D coordinates in (4) and (5). For example, in forest deployments with sensors at different heights in trees, the NeSh model could be applied. However, varying terrain may be difficult to properly model using NeSh, since hills and mountains are typically orders of magnitude larger than other obstructions - a single space constant $\delta$ in (4) may not suffice.

Thirdly, we would expect to see no NeSh model improvement (but no degradation) in environments without obstructions (e.g., empty parking lots) or with extremely small obstructions compared to the path lengths. For example, a completely flat and homogeneous forested environment with link lengths of kilometers, would see shadowing, but few links would cross the same trees. In this case, $\delta / d_{i, j} \rightarrow 0$ and link covariances would also be zero.

Finally, the NeSh model in (5) integrates loss along a line-of-sight (LOS) path between transmitter and receiver. The NeSh model only indirectly incorporates non-LOS paths in the model for shadowing loss. If an LOS path is strongly shadowed, the model effectively assumes that NLOS signals typically arrive with lower power as well 
(due to additional scattering or reflection to go around the obstruction). The normalization by $1 / d_{i, j}^{1 / 2}$ in (5) also helps to diminish the impact of the LOS path as the path length increases (since longer paths tend to have higher NLOS contributions [17]). For longer links, the normalization term decreases the emphasis of the total loss on any one part of the environment. The NeSh model is statistical, so it relies on typical links having such a correlation between NLOS and LOS contributions. However, in strongly reflective environments, e.g., inside an airplane, such an assumption may not be valid, and the NeSh model should not be used.

The model described in this paper is not perfect for all cases, but it does provide a means to incorporate link correlations into network simulation and analysis, which we attempt in the following sections.

\section{Network Connectivity}

It is typically of critical importance to ensure that a multi-hop wireless network is connected. A network can be represented as a graph $\mathcal{G}=(\mathcal{V}, \mathcal{E})$ with vertices $\mathcal{V}$ as the set of all nodes, and a set of directional edges $\mathcal{E}$ containing each transmitter/receiver pair which can communicate. A network is connected if there exists a path between each pair of vertices in its graph. If a network is not connected, it may fail to perform as intended, lacking the capability to transfer data from one part of a network to another. If nodes are mobile, a temporary disconnectivity introduces latency until reconnection. If nodes are stationary, for example in a sensor network, a non-connected network requires a human administrator to go back to repair the sensor network, possibly by moving existing nodes or deploying additional nodes. Since non-connectivity is a major failure, networks are ideally over-provisioned or deployed densely in order to ensure a high probability of connectivity.

In this section, we study the connectivity of particular networks via simulation using both i.i.d. link shadowing and correlated shadowing models. We show that network connectivity in the i.i.d. link shadowing model is overly optimistic. This bias is most severe in robust networks designed to ensure reliable connectivity.

\subsection{Related Research}

Certainly, the vast majority of connectivity research assumes the circular coverage model [13], which allows quick results with relatively simple analysis. Recent work has expanded connectivity analysis by using the i.i.d. log-normal shadowing model. For example, Hekmat and Van Mieghem [10] and Bettstetter and Hartmann [3] studied connectivity in ad hoc networks using the i.i.d. log-normal shadowing model. The model leads in those works to a result that the increase in the variance of shadow fading in the channel also increases the connectivity of the network for the same density of nodes [3]. The results presented in this section show that these connectivity gains are overstated by the use of an i.i.d. link fading model.

\subsection{Methods}

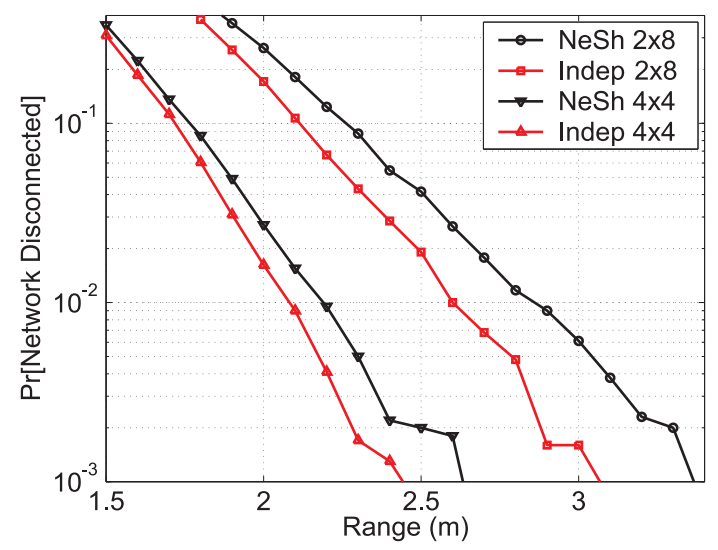

Figure 2. Probability that $4 \times 4$ grid and $8 \times 2$ grid networks are not connected vs. $R_{t h r}$, when using correlated $(-)$ and independent $(-)$ link shadowing models.

Our main assumption in this section is that the ability of a link to communicate is a binary quantization of received power. That is, if $P_{i, j} \geq P_{t h r}$ for some threshold power $P_{t h r}$, then transmitter $i$ can communicate with receiver $j$, and otherwise they cannot communicate. This assumption provides a definition for the common idea of 'range'. We define range $R_{t h r}$ as the distance at which the mean received power in (3) is equal to the threshold power:

$$
R_{t h r}=\Delta_{0} 10^{-\left(P_{t h r}-P_{T}+\Pi_{0}\right) /\left(10 n_{p}\right)} .
$$

As $R_{t h r}$ increases (or equivalently as $P_{T}$ is increased), the connectivity of the network increases.

Here, we assume that transmit powers and threshold powers are identical for each node. In reality, device variations and differing battery levels result in many singledirectional edges. Although these results consider only bi-directional edges, we could address the case of singledirectional links by specifying a random $P_{T}$ and $P_{t h r}$ for each device.

In this paper, we use simulation to generate random graphs which represent random deployments of a multi-hop network. Each deployment has the identical node geometry; because each deployment is in a different place, the radio environment and thus the received power vector $\mathbf{P}$ is different. In the simulation, we set nodes $\mathcal{V}=\{1, \ldots, N\}$, 
select particular coordinates $\left(x_{i}, y_{i}\right)$ for all $i \in \mathcal{V}$, and then perform many independent trials. In each trial we

1. Generate $\mathbf{P}$ from a multivariate Gaussian distribution with mean $\overline{\mathbf{P}}$ from (11) and covariance $C$ from (12).

2. Define the set of edges $\mathcal{E}=\left\{a: P_{a} \geq P_{t h r}\right\}$.

3. Determine whether or not the graph $\mathcal{G}$ is connected.

\subsection{Results}

For simulations, we must set model parameters. We have chosen $\sigma_{d B}=7.0, n_{p}=3.0$, and $\Delta_{0}=1 \mathrm{~m}$; and the measured parameters of $\delta=0.21 \mathrm{~m}$ and $\sigma_{X} / \sigma_{d B}=0.29$ from [2]. In our study, we vary $R_{t h r}$, or equivalently because of (13), vary $\left(P_{T}-\Pi_{0}-P_{t h r}\right)$. This allows us to study the effects of increasing 'range' however that increased range is achieved.

We first consider a sensor network of $N=16$ nodes deployed regularly in a 4 by 4 grid, in a $4 \mathrm{~m}$ by $4 \mathrm{~m}$ area (so that the nodes are spaced each $1.333 \mathrm{~m}$ ). We run $10^{5}$ trials at each value of range $R_{t h r}$ between $1.1 \mathrm{~m}$ and 2.5 $\mathrm{m}$. Figure 2 shows the simulated probability of the network being disconnected, in both i.i.d. link fading and correlated link fading models.

Next we consider the same $N=16$ nodes deployed in a long rectangle, in a 2 by 8 grid, in a $1.5 \mathrm{~m}$ by $10.5 \mathrm{~m}$ area (so that nodes are spaced each $1.5 \mathrm{~m}$ ). This deployment has approximately the same area $\left(16 \mathrm{~m}^{2}\right.$ vs. $15.75 \mathrm{~m}^{2}$ here $)$ and thus the same node density. The longer deployment simulates a sensor network used, for example, in a security system to monitor a border. We run $10^{5}$ trials at each value of range $R_{t h r}$ between $1.8 \mathrm{~m}$ and $3.4 \mathrm{~m}$, and Figure 2 displays the probabilities of non-connectivity in both fading models.

\subsection{Discussion}

For reliable deployments, e.g., with $R_{t h r}=2.2 \mathrm{~m}$ in the square example, the probability of the network being disconnected is $4.1 \times 10^{-3}$ under the i.i.d. link fading model and $9.5 \times 10^{-3}$ under the correlated link shadowing model. The increase of $230 \%$ for the correlated model represents a significant increase in the risk of network non-connectivity. In the rectangular example, for $R_{t h r}=3.0 \mathrm{~m}$, the probability of non-connectivity rises $380 \%$ from $1.6 \times 10^{-3}$ under the i.i.d. link fading model to $6.1 \times 10^{-3}$ under the correlated link shadowing model. We observe that narrow deployment areas magnify the negative effects of link correlation on network connectivity. The results also indicate that as the overall reliability is increased, the over-estimation of connectivity in the i.i.d. link fading model will be increasingly severe.

\section{Sensor Self-Localization}

Lower bounds on sensor self-location estimation have been derived in past research order to provide insight into the effects of system design choices [15, 20, 22, 4, 18]. For example, if the lowest possible localization variance using nodes capable of one measurement modality is too high for the application requirements, a system designer can decide on an alternate measurement modality. As another example, if an implemented estimator achieves a location variance close to the bound, a designer knows that more effort on estimator design will not improve accuracy significantly further.

However, localization bounds reported in the literature have been derived assuming that measurements on different links are independent. If measurements are correlated, such a lower bound would not in fact be rigorous, i.e., the derived lower bound on variance may in fact be higher or lower. In this section, we present the Cramér-Rao bound (CRB) for localization variance when measurements of signal strength are correlated as given by the NeSh model. The CRB is a lower bound on the variance of any unbiased estimator. It is particularly valuable because it does not depend on the algorithm implemented.

\subsection{Derivation}

To derive the Cramér-Rao bound, we assume that coordinates to be estimated, $\boldsymbol{\theta}$, are unknown, where

$$
\boldsymbol{\theta}=\left[x_{1}, \ldots, x_{n}, y_{1}, \ldots, y_{n}\right]^{T},
$$

where $\mathbf{x}_{i}=\left[x_{i}, y_{i}\right]^{T}$ is the coordinate of node $i$. Here, we assume that some devices, nodes $n+1, \ldots, N$ have a priori known coordinate, and thus do not need to be estimated.

Let $\hat{x}_{i}$ and $\hat{y}_{i}$ be unbiased estimators of $x_{i}$ and $y_{i}$. The CRB provides that the trace of the covariance of the $i^{\text {th }}$ location estimate, which we call the location estimation variance bound, satisfies

$$
\begin{aligned}
\sigma_{i}^{2} & \triangleq \operatorname{tr}\left\{\operatorname{cov}_{\boldsymbol{\theta}}\left(\hat{x}_{i}, \hat{y}_{i}\right)\right\}=\operatorname{Var}_{\boldsymbol{\theta}}\left[\hat{x}_{i}\right]+\operatorname{Var}_{\boldsymbol{\theta}}\left[\hat{y}_{i}\right] \\
& \geq\left[\mathbf{F}^{-1}\right]_{i, i}+\left[\mathbf{F}^{-1}\right]_{n+i, n+i}
\end{aligned}
$$

The Fisher information matrix $\mathbf{F}$ for this case is [12],

$$
\begin{aligned}
\mathbf{F} & =\mathbf{F}_{\boldsymbol{\mu}}+\mathbf{F}_{C} \\
{\left[\mathbf{F}_{\boldsymbol{\mu}}\right]_{m, n} } & =\left[\frac{\partial \overline{\mathbf{P}}}{\partial \theta_{m}}\right]^{T} C^{-1}\left[\frac{\partial \overline{\mathbf{P}}}{\partial \theta_{n}}\right] \\
{\left[\mathbf{F}_{C}\right]_{m, n} } & =\frac{1}{2} \operatorname{tr}\left[C^{-1} \frac{\partial C}{\partial \theta_{m}} C^{-1} \frac{\partial C}{\partial \theta_{n}}\right]
\end{aligned}
$$

The derivatives $\frac{\partial \overline{\mathbf{P}}}{\partial \theta_{i}}$ depend on whether the $i$ th parameter is 
an $x$ or $y$ coordinate of a node, and are given in [19] to be:

$$
\begin{gathered}
\frac{\partial \overline{\mathbf{P}}\left(d_{a_{k}}\right)}{\partial x_{m}}= \begin{cases}-\alpha\left(x_{m}-x_{j_{k}}\right) / d_{m, j_{k}}^{2}, & \text { if } m=i_{k} \\
-\alpha\left(x_{m}-x_{i_{k}}\right) / d_{m, i_{k}}^{2}, & \text { if } m=j_{k} \\
0, & \text { otherwise }\end{cases} \\
\frac{\partial \overline{\mathbf{P}}\left(d_{a_{k}}\right)}{\partial y_{m}}= \begin{cases}-\alpha\left(y_{m}-y_{j_{k}}\right) / d_{m, j_{k}}^{2}, & \text { if } m=i_{k} \\
-\alpha\left(y_{m}-y_{i_{k}}\right) / d_{m, i_{k}}^{2}, & \text { if } m=j_{k} \\
0, & \text { otherwise }\end{cases}
\end{gathered}
$$

where $\alpha=\frac{10 n_{p}}{\log 10}$ and distance $d_{m, j_{k}}^{2}=\left\|\mathbf{x}_{m}-\mathbf{x}_{j_{k}}\right\|^{2}$.

\subsection{Discussion of Fisher Information Terms}

We refer to $\mathbf{F}_{\boldsymbol{\mu}}$ as the mean term and $\mathbf{F}_{C}$ as the covariance term of the Fisher information matrix in (15). As the names imply, the mean and covariance terms quantify the information present in the mean and the covariance of the RSS measurements in the network, respectively.

RSS measurements are informative in the mean because the ensemble average RSS measurement in (3) is a function of distance. In CRB analysis under the i.i.d. link fading model, $C=\sigma_{d B}^{2} I$ for identity matrix $I$. Under the NeSh model, the information in the mean term is reduced by the non-diagonal covariance matrix $C$. This will be shown numerically in Section 4.3.

In contrast, the covariance term provides additional information about the coordinates due to the correlations found in the link RSS measurements. In effect, relationships between fading loss measurements on pairs of links in the network will indicate something about the relative geometry of those links. For example, if two links $(i, j)$ and $(i, k)$ both have very high losses $Z_{i, j}$ and $Z_{i, k}$, it may indicate that nodes $j$ and $k$ are in the same relative direction from node $i$. In analyses which use the i.i.d. link fading model, $\mathbf{F}_{C}=0$, and thus no information is gained from the relationships between measurements on pairs of links.

The calculation of $\left\{\frac{\partial C}{\partial \theta_{k}}\right\}_{k}$ is complicated by the size of matrix $C$ and the fact that we require $2 n$ different partial derivatives. We calculate $\frac{\partial C}{\partial \theta_{k}}$ using a finite difference approximation. Writing the covariance matrix as $C(\boldsymbol{\theta})$ to explicitly show it as a function of the coordinates $\boldsymbol{\theta}$,

$$
\frac{\partial C}{\partial \theta_{k}} \approx \frac{C\left(\boldsymbol{\theta}+\epsilon \mathbf{e}_{k}\right)-C(\boldsymbol{\theta})}{\epsilon},
$$

where $\mathbf{e}_{k}$ is the vector of all zeros except for a 1 in the $k^{\text {th }}$ position, and $\epsilon$ is a small positive constant, in this case, we use $\epsilon=10^{-2} \mathrm{~m}$.

\subsection{CRB Comparison in Example Net- works}

To evaluate the relative effect of correlation in the path loss model, we define the relative increase in the standard

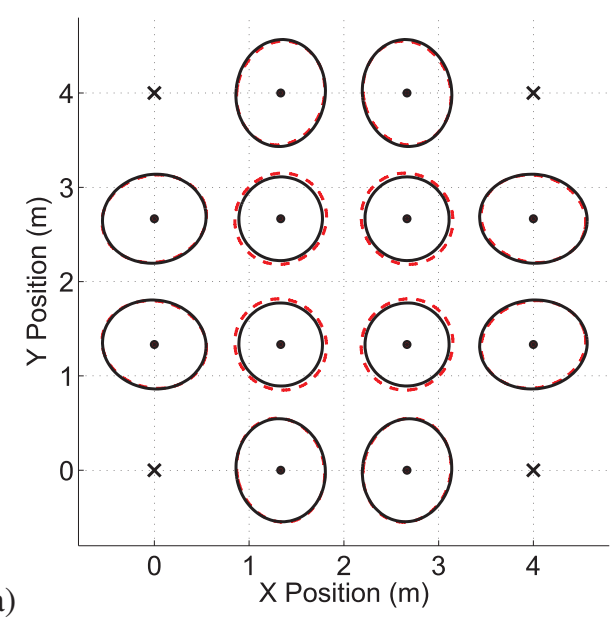

(a)

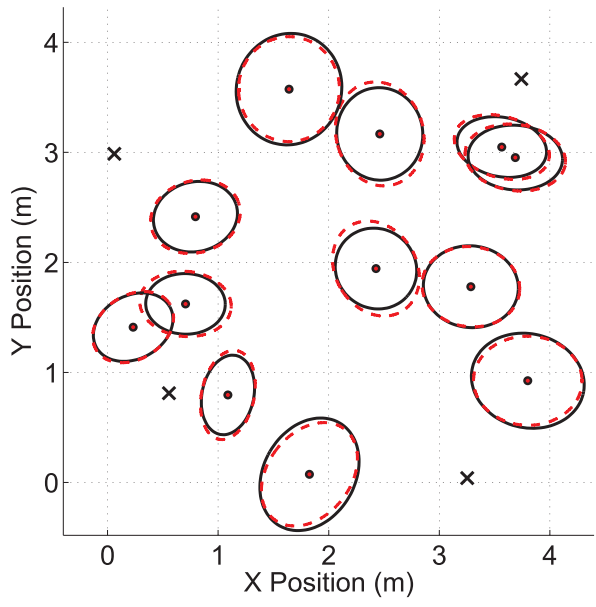

Figure 3. CRB on the one- $\sigma$ uncertainty ellipse when shadowing is correlated $(-)$ and independent (- - - -), for 16-node networks, in (a) grid and (b) random deployments, with actual coordinates of known-location nodes $(x)$ and unknown location nodes $(\bullet)$.

deviation bound compared to the i.i.d. link fading model as

$$
\alpha_{i}=\frac{\sigma_{i}-\tilde{\sigma}_{i}}{\tilde{\sigma}_{i}}
$$

where $\sigma_{i}^{2}$ is the location estimation variance bound from (14) for the case of correlated shadowing using $C$ given in (12), and let $\tilde{\sigma}_{i}^{2}$ denote the variance bound for the case of independent link shadowing (using $C=\sigma_{d B}^{2} I$ ).

We select two particular network geometries for calculation of localization bounds, both in a $4 \mathrm{~m}$ by $4 \mathrm{~m}$ square area. In both cases, we choose the nodes closest to the corners of the square area to be the known-location nodes. That is, the nodes closest to the corners are numbered $N-3, \ldots, N$, and the remaining $n=N-4$ nodes have 
no prior coordinate knowledge.

First, we deploy nodes in a 4 by 4 square grid within the $4 \mathrm{~m}$ by $4 \mathrm{~m}$ area. Nodes are separated by $1.333 \mathrm{~m}, N=16$, and $n=12$. We calculate the CRB in both i.i.d. and correlated link fading models from (14). Figure 3(a) shows the actual location and bound on 1- $\sigma$ covariance ellipse of each node $i=1 \ldots 12$. The $1-\sigma$ covariance ellipse is a useful visual representation of the magnitude and direction of variation of a node's coordinate estimate. The locations of the reference nodes are also shown. For $i=1 \ldots 12$, the values $\alpha_{i}$ are in the range of -0.09 to 0.02 , with an average of -0.024 . This means that on average, the standard deviation bound, when shadow fading correlations are taken into account, decreases by $2.4 \%$.

Next, we generate a random deployment by selecting coordinates independently from a uniform distribution on $[0 \mathrm{~m}, 4 \mathrm{~m}]^{2}$. The actual node locations and the calculated CRB on 1- $\sigma$ covariance ellipse for each node are shown in Figure 3(b). For $i=1 \ldots 12$, the values $\alpha_{i}$ are in the range of -0.13 to 0.03 , with an average of -0.045 . The average standard deviation bound decreases by $4.5 \%$. In general, for random deployments, the decrease in standard deviation bound is more significant than the grid deployment.

\subsection{Discussion and Investigation of Results}

These decreases in the bound may be unexpected, because it is counterintuitive that correlation may improve localization. We might expect that increased link correlation effectively increases the 'noise' level since measurements with additional nodes cannot be used as effectively to 'average-out' fading error. For example, in Figure 1, if an obstruction attenuates both links $(1,3)$ and $(1,4)$, then both link measurements would tend to push nodes 3 and 4 further from node 1. If link measurements were i.i.d., there would be a lower chance of both link measurements $(1,3)$ and $(1,4)$ 'agreeing' nodes 3 and 4 should be further from node 1 .

To investigate further, we momentarily ignore the covariance term $\mathbf{F}_{C}$ and focus solely on the mean term $\mathbf{F}_{\boldsymbol{\mu}}$ in (15). This mean term of the Fisher information matrix should support our above intuition about correlation acting to increase the effective noise level. So, momentarily setting $\mathbf{F}_{C}=0$ in (15), we calculate the bound using $\mathbf{F}=\mathbf{F}_{\boldsymbol{\mu}}$. We call this the NeSh model-based mean term-only bound.

We calculate this NeSh model-based mean term-only bound for the same deployment geometries studied previously in Figure 3. In this case, we compare the $1-\sigma$ covariance ellipses of the NeSh model-based mean term-only bound and of the i.i.d. link fading CRB in Figure 4. The results show clearly that using only the information in the mean term, the lower bound increases when taking into account link correlations. For the grid deployment in Figure 4(a), the values $\alpha_{i}$ are in the range of 0.03 to 0.11 , with an average of 0.080 . This means that on average, when we ignore the information in the covariance term of the Fisher information, the lower bound on standard deviation increases by $8.0 \%$. For the random deployment in Figure 4(b), the same lower bound increases an average of $13.8 \%$. In general random deployments show a more significant increase in lower bound when considering the mean term-only.

This further investigation demonstrates two things:

1. The intuition that link correlations negatively impact localization is true, when estimators only consider the localization information contained in the mean relationship between received power and distance in (3).

2. The use of the information contained in the correlations between links' fading measurements can compensate for the information loss in the mean term, and in fact can reduce localization variances lower than previously thought possible.

These results indicate that estimators which consider correlations between link measurements when estimating node locations will aid the effort to achieve the lowest possible variance. Some localization algorithms have applied statistical learning techniques which explicitly attempt to learn correlations in RSS measurements from training data and then use them to classify or estimate node location $[6,16]$. Other algorithms may be readily adapted to consider link correlations, for example, belief propagation network approaches such as in [11]. However, evaluation of these methods to compare algorithm performance vs. the new bound is beyond the scope of this paper. Future work in RSS-based localization algorithms must investigate approaches to infer location information from link fading correlations.

\section{Radio Tomographic Imaging}

The mechanism which causes correlated link shadowing is detrimental to network connectivity and can be detrimental to sensor self-localization, as discussed in the past two sections. However, there is a benefit gained from the existence of correlated link shadowing, as we discuss in this section. Simultaneous imaging through whole buildings would improve security systems and could save lives in emergency situations. For example, if fire-fighters knew where people were within a building, they could more accurately direct rescue operations, and monitor emergency personnel in building.

If link shadowing is a function of the attenuating properties of the environment in between the transmitter and receiver, then link shadowing measurements can be used to infer those properties. Consider the 20-node network shown in Figure 5. An attenuating object in the building would 


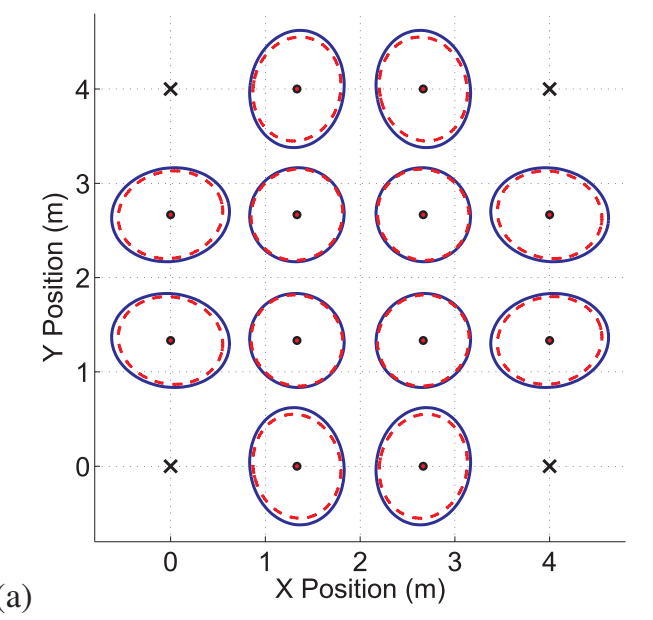

(a)

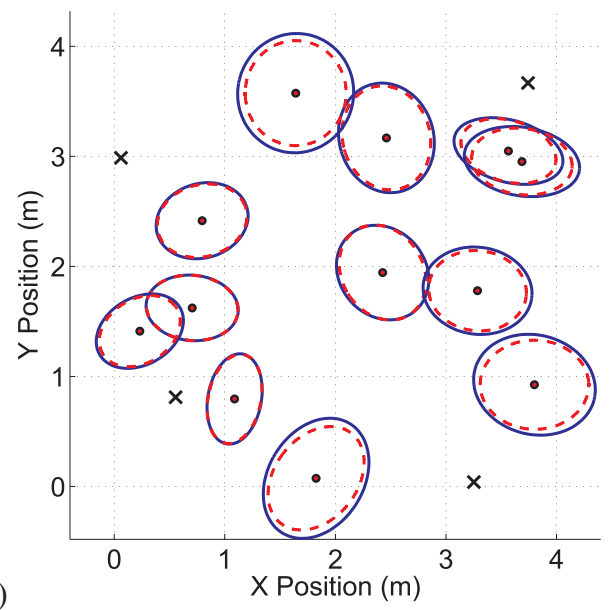

Figure 4. NeSh model 'mean term-only' bound $(-)$ and CRB using the i.i.d. link fading model (- - - ) on localization one- $\sigma$ uncertainty ellipses, for the same (a) grid and (b) random deployments in Figure 3.

tend to increase shadowing loss on multiple links which cross over that object. The inverse perspective on this problem is that high shadowing loss on multiple, intersecting links can be used to infer the location of that attenuating object. By analogy to the medical usage of electromagnetic waves for imaging, we call this method radio tomographic imaging (RTI).

Related work in radar imaging measures scattering, and this imaging method uses transmission. These two wave propagation mechanisms have fundamentally different properties [23, Section 1.10]. In scattering, a wave hits an object and effectively retransmits waves in other directions. The scattered wave measured at the radar device has power on the order of $1 / d^{4}$ (in free space). In transmission, a wave passes through an object and continues in one direc-

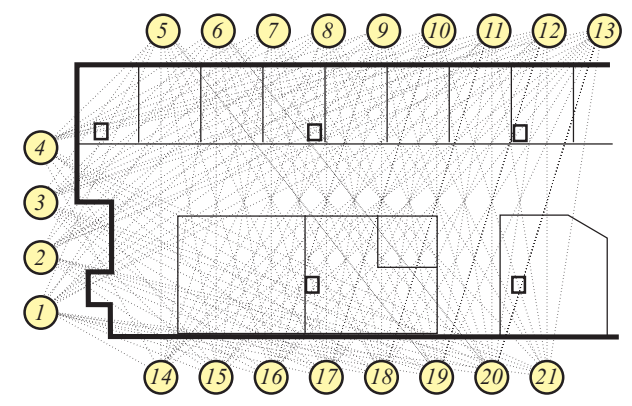

Figure 5 . Nodes measure shadowing on links
covering a building area. Radio tomographic
imaging (RTI) algorithms image the area's
transmission properties.

tion. The transmitted wave loses power due to transmission but arrives at a distant receiver with power on the order of $1 / d^{2}$ (again, in free space). In cluttered environments, both exponents will increase. One fundamental benefit of transmission is that the signal range is approximately the square of that of scattering.

Note that imaging requires correlated shadowing. If link shadowing is independent between links, then there is no chance of being able to image the location of the obstructions in the environment.

Finally, there are certainly many tomographic imaging algorithms, as they have been explored in the literature for multiple purposes over the course of the past decades. The RF tomographic imaging problem introduces physical variations which require special consideration. As such, we don't claim to present an optimal image estimation algorithm, only one reasonable attempt.

\subsection{Motion Imaging Algorithm}

The imaging algorithm proceeds as follows. To compute an image of the motion in the building at time $n$,

1. Difference: Find the link path loss difference, $\nu_{i, j}=$ $P_{i, j}^{a v g}-P_{i, j}$ where $P_{i, j}^{a v g}$ is the past history average received power for link $(i, j)$. Denote $\boldsymbol{\nu}$ as a vector of all $\left\{\nu_{i, j}\right\}_{i, j}$ as in (16).

2. Inverse: Find the weighted least-squared (WLS) error solution for the (pixelated) attenuation field $\mathbf{p}$. The WLS estimator is given by $\hat{\mathbf{p}}=\Pi \nu$, where $\Pi$ is the projection matrix given in (19).

3. Contrast: Convert the real-valued attenuation estimate $\hat{\mathbf{p}}$ into a image vector $\tilde{\mathbf{p}}$ with values in the range $[0,1]$ using the transformation in (20).

These three steps are detailed and justified below. 


\subsection{Difference}

When we image motion, we are not interested in the static attenuation caused by the environment. This static attenuation can be calculated initially, assuming that the nodes are deployed and measuring link losses prior to any motion in the environment. Alternatively, if there is quite a bit of motion during the initial setup, we would expect that an average of path losses measured during a long segment of random motion would reduce the effects of each particular motion. In that case, a system might use a running average of the link losses over a long history to estimate the static attenuation. In any of these cases, we refer to $P_{i, j}^{a v g}$ as the average past history received power on link $(i, j)$. The loss difference $\nu_{i, j}=P_{i, j}^{a v g}-P_{i, j}$ quantifies the current additional loss on link $(i, j)$. Additional loss on this link should be explained by high additional attenuation in the field $p(x)$. As done in Section 2.2, we list unique measured links as $a_{k}=\left(i_{k}, j_{k}\right)$ for $k=1, \ldots, K$ where $K$ is the total number of measured links, and then define

$$
\boldsymbol{\nu}=\left[\nu_{i_{1}, j_{1}}, \ldots, \nu_{i_{K}, j_{K}}\right]^{T}
$$

\subsection{Inverse}

Next, we solve for the pixelated additional loss field,

$$
\mathbf{p}=\left[p\left(\mathbf{y}_{1}\right), \ldots, p\left(\mathbf{y}_{M}\right)\right]^{T}
$$

where $\mathbf{y}_{i}$ is the center coordinate of the $i$ th pixel. The vector $\mathbf{p}$ is the change in attenuation at each pixel of the measured area. We assume that $\mathbf{p}$ is correlated as given in (4).

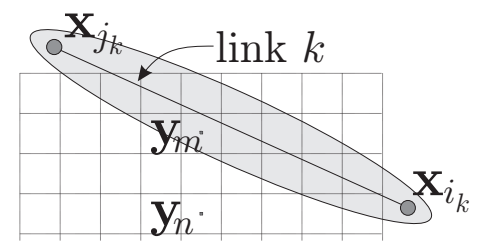

Figure 6. Link $k$ shadowing (between $i_{k}$ and $j_{k}$ ) is a weighted sum of losses in narrow ellipse (foci $\mathbf{x}_{i_{k}}$ and $\mathbf{x}_{i_{l}}$ ). Pixel with center $\mathbf{y}_{m}$ is included, and $\mathbf{y}_{n}$ is not.

Our imaging model is a linearized and discretized version of the link shadow fading equation in (5). Instead of an integral across the attenuation field $p(\mathbf{y})$, we express the link shadowing as a linear combination of the values in vector $\mathbf{p}$, plus noise vector $\mathbf{n}$,

$$
\boldsymbol{\nu}=A \mathbf{p}+\mathbf{n}
$$

We want row $k$ of the transfer matrix $A$ in (17) to be such that the loss on the link $a_{k}$ (between nodes $i_{k}$ and $j_{k}$ ) is a weighted sum of the losses in each pixel between the nodes. Since no pixel center will be exactly on the line from $\mathbf{x}_{i_{k}}$ to $\mathbf{x}_{j_{k}}$, we quantify 'in between' using a narrow ellipse with foci $\mathbf{x}_{i_{k}}$ and $\mathbf{x}_{j_{k}}$. Any pixel $m$ with center coordinate $\mathbf{y}_{m}$ within this ellipse is included in the sum. This is depicted in Figure 6. Specifically,

$$
A_{k, m}=\frac{1}{d_{a_{k}}^{1 / 2}} \cdot\left\{\begin{array}{cl}
1, & \left\|\mathbf{x}_{i_{k}}-\mathbf{y}_{m}\right\|+\left\|\mathbf{x}_{j_{k}}-\mathbf{y}_{m}\right\| \leq d_{a_{k}}+\lambda \\
0, & \text { otherwise }
\end{array}\right.
$$

where $d_{a_{k}}=d_{i_{k}, j_{k}}=\left\|\mathbf{x}_{i_{k}}-\mathbf{x}_{j_{k}}\right\|, \lambda$ is the parameter which determines the ellipse width, and $d_{\min }$ is a minimum link distance. The normalization by $d_{a_{k}}^{1 / 2}$ in (18) parallels the normalization term in the model (5).

We set $d_{\min }$ much less than the dimension of the sensed area. Very short link distances, $d_{a_{k}}<d_{\min }$, tend to be on links which do not impinge on the area to be imaged (since sensors are outside of the area). This limitation avoids considering these uninformative link measurements.

Since pixels are correlated, it is critical to use that correlation in the image estimator. Thus we choose to use the regularized weighted least-squared error (WLS) estimator,

$$
\begin{aligned}
& \hat{\mathbf{p}}=\Pi \boldsymbol{\nu} \\
& \Pi=R A^{T}\left(A R A^{T}+\sigma_{K}^{2} I_{K}\right)^{-1}
\end{aligned}
$$

The term $\sigma_{K}^{2}$ is a regularization constant, $I_{K}$ is the $K \times K$ identity matrix, $R$ is the correlation matrix with $(i, j)$ th element $R_{i, j}=R_{p}\left(\left\|\mathbf{y}_{i}-\mathbf{y}_{j}\right\|\right)$ given in (4), and $A$ is the transfer matrix from (17). The regularization term $\sigma_{K}^{2} I_{K}$ makes the estimator robust to any rank-deficiency in transfer matrix $A$. Rank-deficiency should be expected, both because there can be more pixels than link measurements, and even if not, we have not ensured that each pixel's attenuation can be uniquely determined.

Note that computational complexity is small enough to be implemented in real time. The projection matrix $\Pi$ is calculated only once. The 'inverse' step requires only the multiplication of $\Pi$ and vector $\nu$.

\subsection{Contrast}

In the final step, we adjust the real-valued pixels $\hat{\mathbf{p}}$ to a intensity in $[0,1]$ in a way that will be meaningful to a user. Rather than simply linearly scale the values of $\hat{\mathbf{p}}$ to fit within $[0,1]$, we want to help the user visualize which pixels show statistically significant attenuation. In this paper, we do this by setting a threshold value of attenuation which has a low probability of being measured in static conditions. We then display those pixels higher than the threshold attenuation value to a intensity value above 0.5 , and those pixels below the threshold to an intensity below 0.5 .

Specifically, our threshold is $c \sigma_{p}$, where $\sigma_{p}$ is the standard deviation of all values of $\hat{\mathbf{p}}$ in the startup history, and 
Table 1. Motion Experiment Timeline

$\begin{array}{ll}\text { 19:00:08-19:01:02 } & \text { Open door, walk to, then stand in, } \\ & \text { SW corner. } \\ \text { 19:01:03-19:02:02 } & \text { Walk to, then stand in, SE corner. } \\ \text { 19:02:03-19:03:08 } & \text { Walk to, then stand in, NE corner. } \\ \text { 19:03:09-19:04:03 } & \text { Walk to, then stand in, NW corner. } \\ \text { 19:04:04 } & \text { Exit room. }\end{array}$

$c$ is a positive constant. Since link losses $\boldsymbol{\nu}$ are zero-mean multivariate Gaussian (in $\mathrm{dB}$ ) and $\hat{\mathbf{p}}$ is a linear combination of $\boldsymbol{\nu}$, pixel values $\hat{\mathbf{p}}$ will also be zero-mean and Gaussian. Thus the false alarm probability, i.e., that $\hat{p}\left(\mathbf{y}_{i}\right)>c \sigma_{p}$ when the environment is static, is approximately $\mathrm{Q}(c)$, where $\mathrm{Q}(\cdot)$ is the complementary CDF of a standard normal random variable, $\mathrm{Q}(x)=\frac{1}{2}[1-\operatorname{erf}(x / \sqrt{2})]$. For example, in the experiment described in Section 5.5, we use $c=2.8$, which makes the false alarm probability approximately $0.25 \%$.

We also want to scale pixel values so that an intensity of 1 indicates the highest pixel value. We do this by using the following (nonlinear) scaling function:

$$
\tilde{\mathbf{p}}=1-\mathrm{Q}\left(\frac{\hat{\mathbf{p}}-c \sigma_{p}}{a}\right)
$$

where we choose a scaling constant $a$ based on the maximum value of $\hat{\mathbf{p}}$, which we denote $p_{\max }=\max \hat{\mathbf{p}}$,

$$
a=\max \left[\epsilon_{a},\left(p_{\max }-c \sigma_{p}\right) / b\right]
$$

where $\epsilon_{a}$ and $b$ are predetermined positive constants. We must ensure that $a>0$ so that (20) does not have a divide-by-zero, and thus (21) assigns it a minimum of $\epsilon_{a}$. The result of (21) is to ensure that when some pixels have $p\left(\mathbf{y}_{i}\right)>c \sigma_{p}$, that the maximum attenuation in the image is always displayed with intensity value of $\mathbf{Q}(b)$. For example, we use $b=5$ in Section 5.5, so that this maximum intensity is very close to 1 .

\subsection{Experiment}

We use an unoccupied $5 \mathrm{~m}$ by $5 \mathrm{~m}$ room in the Warnock Engineering Building on the University of Utah campus. All walls are interior walls, and mica2 sensors are placed outside of the room, about $0.5 \mathrm{~m}$ from each wall, as shown in Figure 7(f). Admittedly, an empty room is a friendly environment for RTI, and future tests must verify that RTI can work properly in average cluttered building environments.

Each sensor runs a TinyOS program to record the RSS and id number from any messages received from its neighbors. It also transmits (at $915 \mathrm{MHz}$ ), each half second, a message containing its id and the (id, RSS) measurement pairs which it recorded in the past half second. A laptop

\begin{tabular}{|c|c|}
\hline Variable Nam & Description \\
\hline & periment Description \\
\hline$N=20$ & Number of sensors \\
\hline$K=190$ & Number of links measured \\
\hline$M=121$ & Number of pixels \\
\hline$P_{i, j}^{a v g}$ & Average $P_{i, j}$ prior to $19: 00: 08$ \\
\hline$\sigma_{p}$ & $\begin{array}{l}\text { Std. dev. of pixel values } \mathbf{p} \text { prior to } \\
\text { 19:00:08 }\end{array}$ \\
\hline Chan & Model Parameters from [2] \\
\hline$\delta=0.21 \mathrm{~m}$ & Attenuation field correlation distance \\
\hline$\frac{\sigma_{X}^{2}}{\sigma_{d B}^{2}}=0.29$ & Shadowing variance ratio \\
\hline & Algorithm Parameters \\
\hline$\lambda=0.2 \mathrm{~m}$ & Ellipse 'width' parameter \\
\hline$d_{\min }=2 \mathrm{~m}$ & Minimum link length \\
\hline$\sigma_{K}^{2}=3$ & Regularization constant \\
\hline$c=2.8$ & \# of $\sigma_{p}$ for contrast threshold \\
\hline$b=5$ & Maximum contrast parameter \\
\hline$\epsilon_{a}=0.1$ & Minimum contrast scale factor \\
\hline
\end{tabular}

Table 2. RTI Experiment Settings

connected to a listening node records and time-stamps all packets transmitted by the sensors. The laptop is also connected to a video camera which records time-stamped images from within the room so that the nature of activity is known at each second.

To reduce missing data (due to interference), we average the two measurements over time on a link $(i, j)$ and the two reciprocal channel measurements on link $(j, i)$. Thus, each second, there is one measurement for the bi-directional link between nodes $i$ and $j, P_{i, j}$. If there were no measurements to average, we set $P_{i, j}=P_{i, j}^{a v g}$.

The sensors are turned on and left running for about 40 minutes. This is the 'startup period' used to initialize $\left\{P_{i, j}^{a v g}\right\}_{i, j}$ and $\sigma_{p}$. Beginning at time 19:00:08, as detailed in Table 1, a person walks into the room, walks to and then stands in each corner, each for one minute, and finally leaves the room.

We follow the algorithm steps outlined above to calculate the image intensity vector $\tilde{\mathbf{p}}$ each second, using the constants listed in Table 2 and display images. In Figure 7(a-e), images recorded exactly one minute apart are shown. The first four images are those at 19:00:23, 19:01:23, 19:02:23, and 19:03:23, within 20 seconds after the movement to the SE, SW, NW, and NE corners, respectively. The final image shown in Figure 7(e) is taken at 19:04:23, about 20 seconds after the person left the room.

\subsection{Discussion}

From Figure 7, we note positive results and opportunities for future improvement. The results show that the extra at- 


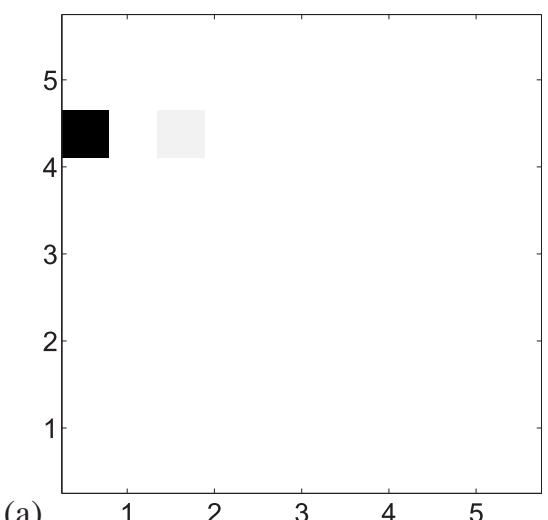

(a)
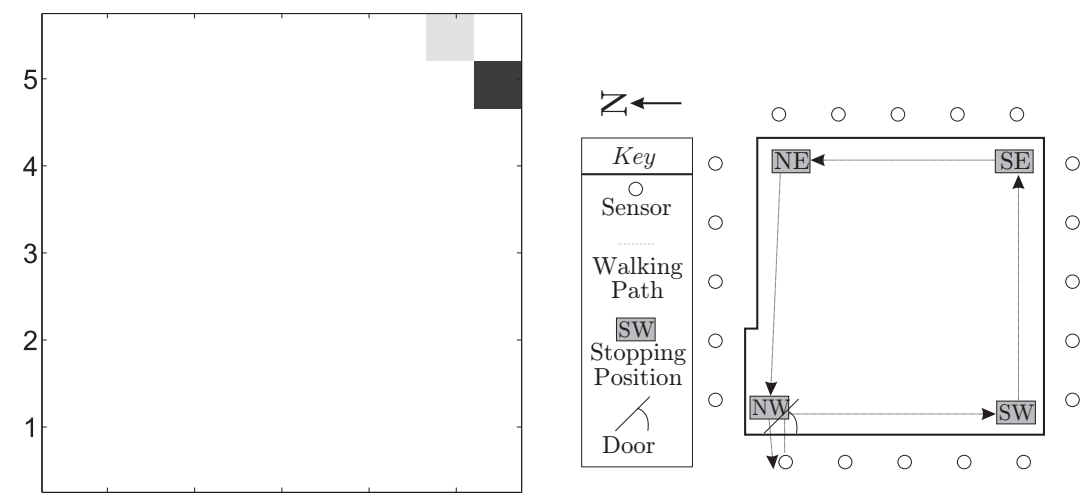

(b)

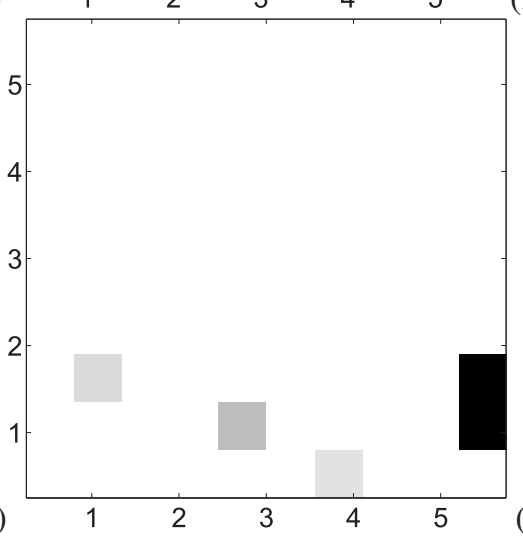

(f)

(c)

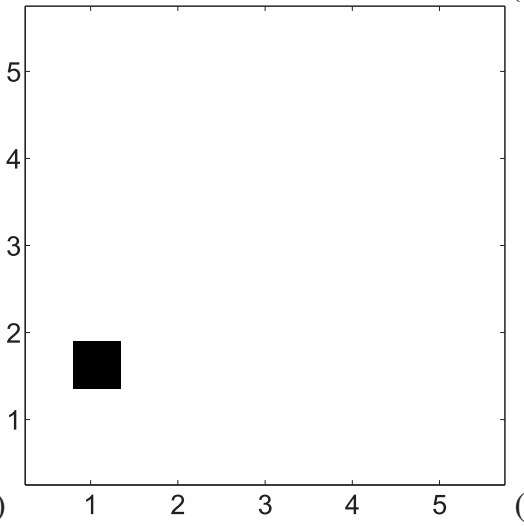

(d)

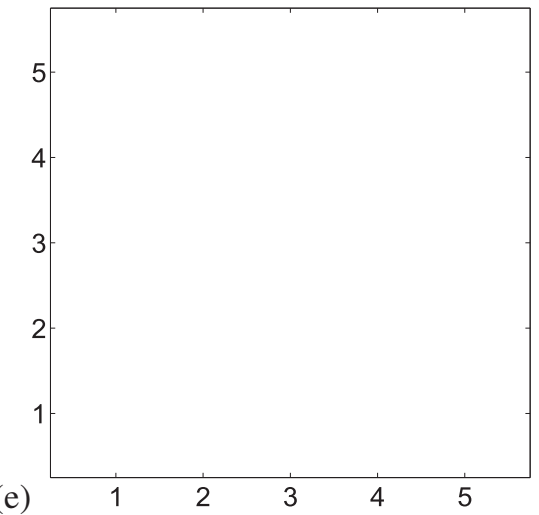

Figure 7. Radio tomographic images for times and person locations (a) 19:02:23 in NE corner, (b) 19:01:23 in SE corner, (c) 19:03:23 in NW corner, (d) 19:00:23 in SW corner, and (e) 19:04:23 when person was out of room. In (f) is an area map with diagram of the experiment.

tenuation caused by a person in a room can be both detected and located. When no motion exists, images are almost always empty, with $\tilde{\mathbf{p}} \approx 0$. While a person is stationary in the room, there is noticeable change, and the darkest pixels indicate the general location of that extra attenuation. Finally, Figure 7 does not show calculated images during a person's movement, but we note that: (1) the image clearly displays that motion exists with multiple dark pixels, but (2) the image does not accurately locate that motion. We suppose that since the sensors, as programmed, do not measure the link losses simultaneously, the differences are enough to confuse the result.

Further research is still warranted. Figure 7(a) shows pixels with medium intensity at locations other than the SE corner of the room. Periodically, such random effects are seen in the images calculated while a person was stationary. These undesired effects may be caused by multipath fading, since a person acts not only as an attenuator, but also as a reflector and scatterer. These effects may be smaller in magnitude than the LOS effects, but because the RSS measurements are narrowband and subject to narrowband fading, there can be significant loss difference in other links besides those crossing the pixel where the person is standing. We believe both wideband RSS measurements and more extensive time-averaging will be useful to reduce these effects.

Finally, we used relatively large pixels in this implementation, each $0.25 \mathrm{~m}^{2}$ in area. This is influenced by the fact that with 20 sensors there are 190 link measurements, and with the given pixel size, we have 121 pixels in the image. We expect that we will require more link measurements than pixels in order to reduce the 'noise' effects caused by narrowband fading. Moreover, we can't expect RF-based tomography to have the same resolution as $\mathrm{x}$-ray tomography - the wavelength at $900 \mathrm{MHz}$ is $33 \mathrm{~cm}$, which is a limiting factor for RTI.

Note that the number of links will likely increase at the same rate as the number of pixels as the area size increases. If sensors are deployed with constant $\Delta x$ spacing between them around the perimeter of a square area with side length $L$, both the number of pixels and the number of links increase as $\mathcal{O}\left(L^{2}\right)$. Thus we can expect constant resolution as we apply RTI to larger areas. 


\section{Conclusion}

This paper demonstrates quantitative effects of the realworld phenomenon of correlated shadowing on links, in connectivity, localization, and in radio tomographic imaging. The results indicate that: (1) reliable connectivity will be much more difficult than predicted by i.i.d. link models, (2) RSS-based localization algorithms must use link correlations in coordinate estimation to reduce variance, and (3) link correlations can be used to infer the location of motion in an environment.

Generally, the three topics only touch the surface of the effects on sensor networks. For example, while connectivity was considered, coverage was not, yet coverage and connectivity are closely related [24]. Sensors deployed to listen for RF signals would have their coverage area affected by shadowing correlation. Moreover, if the propagation in other media, such as sound and light, are similarly correlated by obstructions in the environment, then acoustic and video sensor networks would have coverage areas affected by correlation. Further, exposure problems, such as finding the minimal exposure path, will be affected. Finally, the choice of physical layer model will affect analysis of network capacity, cooperative communication schemes, and energy consumption. Analysis can be improved by applying more accurate shadowing models such as the NeSh model applied in this paper.

Future research must also test and verify the NeSh model in different environments, at different frequencies, and at different network densities, in order to verify the model and to provide measured $\sigma_{X}^{2}$ and $\delta$ for common cases of multihop network deployments.

\section{Acknowledgements}

The authors wish to thank Steven S. King for his aid in conducting the measurement experiments in Section 5.

\section{References}

[1] Sensing and Processing Across Networks (SPAN) Lab website. http://span.ece.utah.edu.

[2] P. Agrawal and N. Patwari. Correlated link shadow fading in multi-hop wireless networks. IEEE Trans. Wireless Commun. Submitted Nov. 2007, [Online] http://span.ece.utah.edu/pmwiki/pmwiki. php?n=Main. Archives.

[3] C. Bettstetter and C. Hartmann. Connectivity of wireless multihop networks in a shadow fading environment. Wirel. Netw., 11(5):571-579, 2005.

[4] A. Catovic and Z. Sahinoglu. The Cramér-Rao bounds of hybrid TOA/RSS and TDOA/RSS location estimation schemes. IEEE Commun. Lett., 8(10):626-628, Oct. 2004.
[5] G. D. Durgin. Space-Time Wireless Channels. Prentice Hall PTR, 2002.

[6] B. Ferris, D. Hahnel, and D. Fox. Gaussian processes for signal strength-based location estimation. In Proc. Robotics Science and Systems, 2006.

[7] M. Gudmundson. Correlation model for shadow fading in mobile radio systems. IEE Electronics Letters, 27(23):2145 -2146, 7 Nov. 1991.

[8] P. Gupta and P. R. Kumar. The capacity of wireless networks. IEEE Trans. Info. Theory, 46(2):388-404, Mar. 2000.

[9] H. Hashemi. The indoor radio propagation channel. Proc. IEEE, 81(7):943-968, July 1993.

[10] R. Hekmat and P. V. Mieghem. Connectivity in wireless adhoc networks with a log-normal radio model. Springer Mobile Networks and Applications, 11:351-360, April 2006.

[11] A. T. Ihler, J. W. Fisher III, and R. L. Moses. Nonparametric belief propagation for self-calibration in sensor networks. In IEEE ICASSP 2004, volume 3, pages 861-864, May 2004.

[12] S. M. Kay. Fundamentals of Statistical Signal Processing. Prentice Hall, New Jersey, 1993.

[13] D. Kotz, C. Newport, and C. Elliott. The mistaken axioms of wireless-network research. Technical Report TR2003-467, Dept. of Computer Science, Dartmouth College, July 2003.

[14] B. Krishnamachari. Networking Wireless Sensors. Cambridge University Press, Cambridge UK, 2005.

[15] R. L. Moses, D. Krishnamurthy, and R. Patterson. An auto-calibration method for unattended ground sensors. In ICASSP, volume 3, pages 2941-2944, May 2002.

[16] X. Nguyen, M. I. Jordan, and B. Sinopoli. A kernel-based learning approach to ad hoc sensor network localization. ACM Trans. Sen. Netw., 1(1):134-152, 2005.

[17] K. Pahlavan, P. Krishnamurthy, and J. Beneat. Wideband radio propagation modeling for indoor geolocation applications. IEEE Comm. Magazine, 36:60-65, April 1998.

[18] N. Patwari, J. Ash, S. Kyperountas, R. M. Moses, A. O. Hero III, and N. S. Correal. Locating the nodes: Cooperative localization in wireless sensor networks. IEEE Signal Process., 22(4):54-69, July 2005.

[19] N. Patwari and A. O. Hero III. Signal strength localization bounds in ad hoc \& sensor networks when transmit powers are random. In Fourth IEEE Workshop on Sensor Array and Multichannel Processing (SAM'06), July 2006.

[20] N. Patwari, A. O. Hero III, M. Perkins, N. Correal, and R. J. O'Dea. Relative location estimation in wireless sensor networks. IEEE Trans. Signal Process., 51(8):2137-2148, Aug. 2003.

[21] T. S. Rappaport. Wireless Communications: Principles and Practice. Prentice-Hall Inc., New Jersey, 1996.

[22] A. Savvides, W. Garber, S. Adlakha, R. Moses, and M. B. Srivastava. On the error characteristics of multihop node localization in ad-hoc sensor netwoks. In 2nd Intl. Workshop on Inform. Proc. in Sensor Networks, April 2003.

[23] W. L. Stutzman and G. A. Theile. Antenna Theory and Design. John Wiley \& Sons, 1981.

[24] H. Zhang and J. C. Hou. Maintaining sensing coverage and connectivity in large sensor networks. Ad Hoc \& Sensor Wireless Networks (Old City), 1:89-124, March 2005. 